\title{
Assessment of Climatic and Anthropogenic Controls on Bridge Deck Drainage and Sediment Removal
}

\author{
Alexander Michalek ${ }^{1}$, Admin Husic ${ }^{2}, * \mathbb{E}$, Joshua Roundy ${ }^{2}$ and Amy T. Hansen ${ }^{2}$ \\ 1 Department of Civil and Environmental Engineering, University of Iowa, Iowa City, IA 52242, USA; \\ atmichalek@uiowa.edu \\ 2 Department of Civil, Environmental and Architectural Engineering, University of Kansas, \\ Lawrence, KS 66045, USA; jkroundy@ku.edu (J.R.); amy.hansen@ku.edu (A.T.H.) \\ * Correspondence: ahusic@ku.edu
}

check for updates

Citation: Michalek, A.; Husic, A.; Roundy, J.; Hansen, A.T. Assessment of Climatic and Anthropogenic Controls on Bridge Deck Drainage and Sediment Removal. Water 2021, 13, 3556. https://doi.org/10.3390/ w13243556

Academic Editor: Chia-Ren Chu

Received: 10 November 2021 Accepted: 10 December 2021 Published: 12 December 2021

Publisher's Note: MDPI stays neutral with regard to jurisdictional claims in published maps and institutional affiliations.

Copyright: (c) 2021 by the authors. Licensee MDPI, Basel, Switzerland. This article is an open access article distributed under the terms and conditions of the Creative Commons Attribution (CC BY) license (https:// creativecommons.org/licenses/by/ $4.0 /)$.

\begin{abstract}
Bridge deck drainage is essential to prevent hydroplaning and maintain safety along major roadways. With projected changes in climate, current designs may not be sufficient and a better understanding of the primary controls (climate, bridge deck, and inlet design) on the hydraulic efficiency and sediment removal of drainage systems is needed to maintain public safety. To evaluate the controls on hydraulic drainage efficiency, 576 controlled laboratory experiments were conducted testing grate type (rectangular bar vs. curved vane) and downspout configuration (square vs. circular and $20 \mathrm{~cm}$ vs. $25 \mathrm{~cm}$ ) across a range of flow rates, cross slopes, and longitudinal slopes. An additional 144 sediment erosion experiments were performed to identify controls on the removal of sediment. Hydraulic testing indicated that inflow driven by climate is a primary control on drainage efficiency and spread of water on a roadway. For anthropogenic controls, downspout opening size was found to be the primary control followed by longitudinal slope. Sediment removal results indicated that inflow regime and grate type were the primary controls on the sediment removal rate. Given that inflow, driven by climate, is a control on both hydraulic and sediment removal performance, hydraulic engineers should consider forecasted changes in rainfall intensity in their present-day drainage designs. We provide design guidance and discussion for developing a proactive approach to hydraulic infrastructure in the face of future climate uncertainty.
\end{abstract}

Keywords: bridge deck drain; experimental model; design; climate; debris; sediment transport

\section{Introduction}

Roadway systems are important aspects of the modern world and allow for the interaction between people and communities that prompts economic growth and stability [1]. To protect this function and ensure trust within the public, traffic safety is a top priority for engineers. Traffic safety can be compromised by large precipitation events as the accumulation of water within a roadway increases the risk of hydroplaning, potentially causing the loss of vehicle control and human life [2]. To prevent hydroplaning, drainage systems are designed to convey stormwater runoff away from the roadway surface and limit the spread of accumulated water into traffic lanes, highlighting the importance of drainage system design when considering roadway infrastructure.

Roadways and bridge systems are in crucial need for repair with the condition of over $43 \%$ of public roadways in America rated as poor or mediocre [3]. The adequacy of current infrastructure systems to safely convey traffic during rainfall events will only worsen as changes to climate, such as extreme precipitation, temperature, and windstorm events, increase infrastructure stress [4-8]. In particular, climate change will stress roadway drainage by increasing rainfall intensities in much of the world, resulting in more runoff and debris flow [8] and increasing the susceptibility for clogging. There is potential that current drainage system designs lack adequate capacity to handle these forecasted stressors, lowering the safety for commuters on roadways [4,5]. Preparing for these issues now is 
crucial to reducing future risks due to climate change [9]. With the current need for updated infrastructure, the opportunity is present to review and update current roadway drainage systems and designs to better prepare for the future.

The appropriate choices regarding how to update roadway or bridge infrastructure depend upon the variables that control ponding of water. For example, roadway design, drainage inlet design, and inlet clogging status all affect the potential for water buildup and hydroplaning. Roadway drainage first conveys sheet flow laterally to the curb where it channelizes as gutter flow and is conveyed longitudinally toward drainage inlets (Figure 1). By capturing and removing gutter flow, drainage inlets limit the spread of water $(T)$ across the deck. Drainage system design is governed by the goal to contain the spread of water within the shoulder area and out of driving lanes [10]. To determine the number and spacing of inlets required for necessary drainage, the projected magnitude of gutter flow and capacity of the inlets to intercept flow are considered [11,12]. Additionally, bridge drainage inlets are often plagued by debris accumulation (Supplementary Materials Table S1), which reduce their ability to convey runoff from the roadway making it also important to understand the impact of design parameters on debris movement.

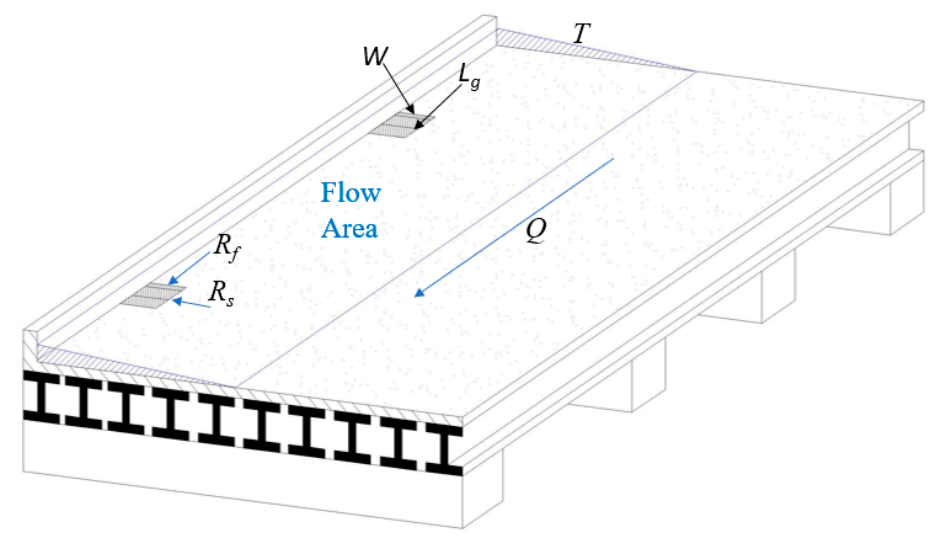

Figure 1. Conceptual Diagram of a bridge deck with drainage inlets depicting terms of inlet width $(W)$, inlet length $\left(L_{g}\right), Q$, frontal flow ratio $(R f)$, side flow ratio $(R s)$, and width of flow $(T)$.

Recent literature [2,13] on bridge deck drainage systems has focused on the implementation and assessment of experimentally derived design equations. We aim to understand the controls on drainage design from a broader perspective by comparing different combination of anthropogenic controls (bridge deck and inlet design parameters) and climate controls to provide holistic design guidance. Additionally, we analyze the impact of anthropogenic and climatic controls on sediment removal rate of drainage inlets which, to our knowledge, has not been examined before. To achieve our aim, we use a scaled physical model to determine the primary controls on bridge deck drainage efficiency and drainage inlet sediment removal by varying climate drivers, bridge deck design parameters, and drainage inlet design parameters. We develop two primary experimental setups. The first experimental setup examines hydraulic efficiency and spread for multiple configurations of gutter flow, roadway slope, and inlet designs to compare standard design performance metrics for design guidance. The second experimental setup tested sediment removal rates with multiple configurations of gutter flows, bridge slopes, and grate designs in order to develop guidelines for preventing the commonly observed field issue of inlet clogging (Supplementary Materials Table S1). In each set of experiments, design factors were examined through related experimental variables. Climate is examined through inflow settings, bridge deck design by model slope, and inlet design by downspout opening size and grate type. The results of this study provide guidance toward effective and proactive approaches to ensuring new and existing bridge infrastructure and drainage systems continue to meet traffic safety mandates in the face of a changing climate. 


\section{Materials and Methods}

\subsection{Laboratory Experimental Setup}

The primary method to test the goals of the study was through the use of a physical scale model as is commonly used in studies [2,10-13] related to roadway drainage. The use of experimental model allowed for directly testing the impact of longitudinal slope, cross slope, inflow, and inlet type on the removal of gutter flow from a roadway surface and width of water on the roadway. Each of these parameters are important for drainage design. An experimental model provides flexibility as multiple combinations of variables can be tested and it allows us to control the quality and quantity of data needed for our analysis. This was especially crucial for analysis of sediment removal rates related to grate design where limited literature exists.

\subsubsection{Hydraulic Efficiency Experiment}

The first set of experiments was conducted to quantify hydraulic efficiency, $E$, or the fraction of total gutter flow captured by an inlet as defined by the following equation [10]:

$$
E=\frac{Q_{c}}{Q}
$$

where $Q_{c}$ is the flow captured by the inlet $\left(\mathrm{m}^{3} / \mathrm{s}\right)$ and $Q$ is the total gutter flow in the channel $\left(\mathrm{m}^{3} / \mathrm{s}\right)$. Gutter flow, as described in Figure 1, is defined by the following relationship in design [11,12]:

$$
Q=\left(\frac{k_{g}}{n}\right) S_{x}^{1.67} S^{0.5} T^{2.67}
$$

where $Q=$ total gutter flow rate, $k_{g}=$ gutter flow unit conversion factor ( 0.375 for SI units), $n=$ Manning's roughness coefficient, $T=$ width of flow, $S_{x}=$ cross slope of the roadway, and $S$ = longitudinal slope of the roadway. This parameter is measured in the experiment but shown here for background on the relationship between experimental variables.

Hydraulic efficiency experiments were conducted in a 1:9 scale laboratory model, representing a $6.4 \mathrm{~m}$ single lane highway and shoulder on a bridge deck (Figure 2a). The model was suspended in a $10.16 \mathrm{~m}$ long, $0.91 \mathrm{~m}$ wide, and $1.52 \mathrm{~m}$ deep flume with cables attached to supports across the top of the flume. The width of the model was $0.71 \mathrm{~m}$ with a length of $10.16 \mathrm{~m}$. The cable supports allowed for full adjustability of longitudinal slope, $\mathrm{S}$, and cross slope, $\mathrm{S}_{\mathrm{x}}$, across the range of 0.5 to $4 \%$ and 2 to $6 \%$, respectively.

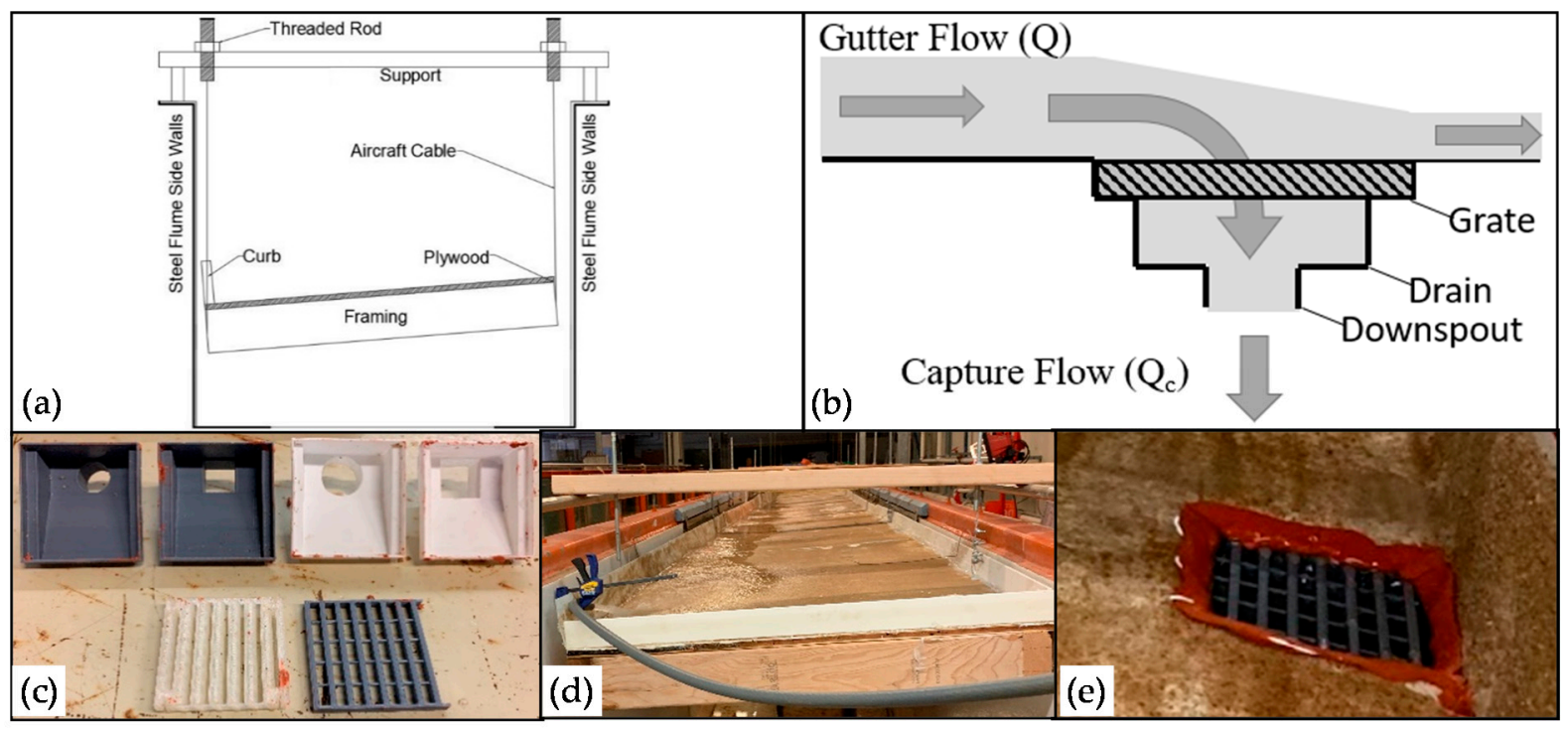

Figure 2. (a) Cross-sectional and (b) side profile views of experimental bridge deck set-up with dimensions, layout, and materials used. Images of (c) 3-D printed inlet (circular and square) and grate (bar and vane) designs, (d) above-deck inflow setup, and (e) placement of inlet on deck surface. 
A single opening in the deck gutter was created to test grate inlet designs (Figure $2 b$ ). The inlets and grates were scaled using a modeling software and 3-D printed for precision and control of design (Figure 2c). The four inlet types printed for this study simulated $0.61 \mathrm{~m}$ square openings. Bar and vane grates were scaled and printed following design drawings provided by Kansas (KDOT) [14] and Illinois (ILDOT) [15] Departments of Transportation. Square and circular downspout shapes were tested for two widths/diameters $(20 \mathrm{~cm}$ and $25 \mathrm{~cm}$ ). Upstream inflow to the model was provided by a hose with a calibrated flow meter (Tuthill TT10PN) (Figure 2d) with an accuracy of $+/-1.00 \%$. The hose was placed against the curb of the deck facing downslope to immediately simulate channelized gutter flow. Measurement tapes were attached $0.76 \mathrm{~m}$ upstream of the inlet to measure the spread of water. To represent the roughness of bridge deck surfaces, the standard Manning's n (0.016) used by KDOT [14] was scaled to 0.012 by application of a length ratio relationship. Sand particles $(0.5 \mathrm{~mm}$ silica) with an equivalent grain roughness were adhered to the deck. Flow captured by the inlet (Figure 2e) was measured using a scale (Ohaus SD75) and collection bin placed beneath the deck. The scales used in the experiment are professionally calibrated every year and a check was performed before experimentation. The tolerance of each scale was $50 \mathrm{~g}$. The system was automated with solenoid valves to drain the collection bins between experiments. A full breakdown of experimental model and materials can be found in Supplementary Materials Table S2.

The procedure for measuring $E$ dependence on cross slope, longitudinal slope, grate type, and downspout configuration was to run the experimental series listed in Figure 3 at low $\left(0.7 \mathrm{~m}^{3} / \mathrm{h}\right)$, medium $\left(1.0 \mathrm{~m}^{3} / \mathrm{h}\right)$, and high $\left(1.6 \mathrm{~m}^{3} / \mathrm{h}\right)$ inflow regimes with three replicates per configuration. For each trial, the deck surface was wetted by inflow for $30 \mathrm{~s}$ prior to data collection to remove the influence of surface material on water spread. Each experimental run lasted three minutes. Starting and ending captured weights and spreads were recorded for each run. Analysis of the results from the hydraulic efficiency experiment compared parameters of efficiency and spread.

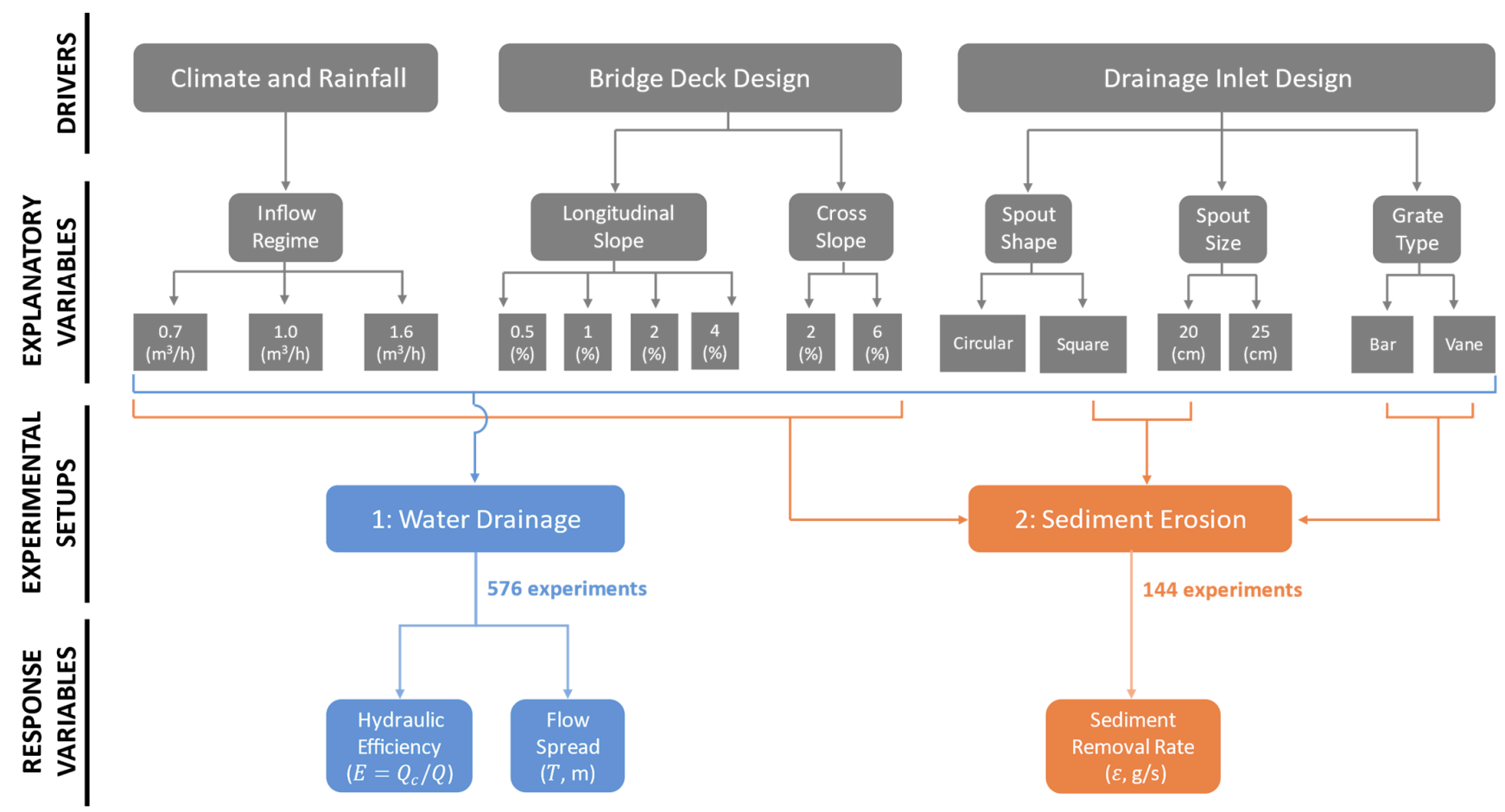

Figure 3. Experimental design for water drainage and sediment erosion. Note: sediment erosion experiments are performed only on $20 \mathrm{~cm}$ square downspout configurations. Triplicates were performed for each combination of variables. 


\subsubsection{Sediment Removal Experiment}

The second set of experiments was conducted to measure inlet sediment removal rate based on grate type (vane or bar) and flow intensity. The experimental setup for hydraulic efficiency was modified to extend experiments from 3 to $10 \mathrm{~min}$, allowing for measurable erosion to occur. At the inlet, a cohesive sediment mixture was packed underneath the grate to simulate field conditions (Figure 4).

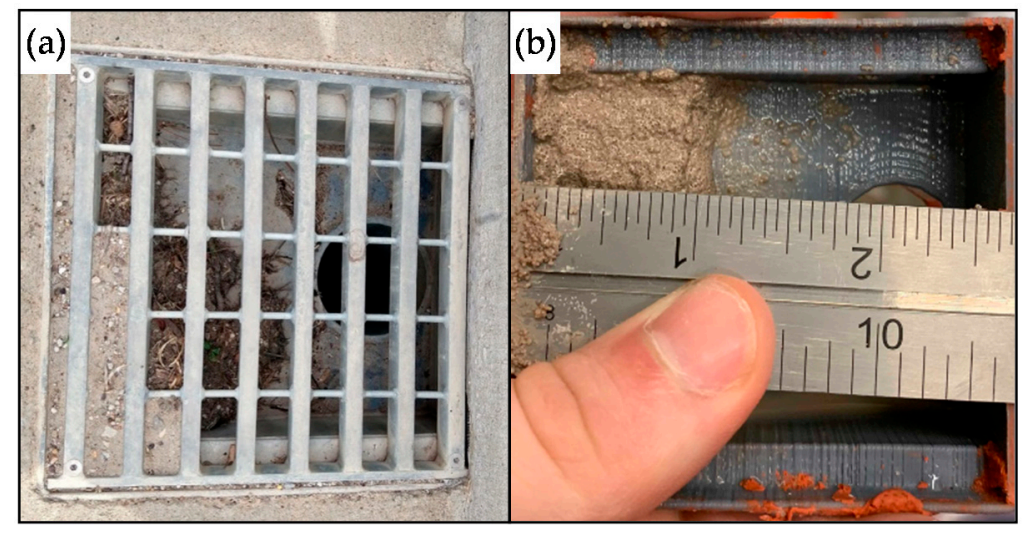

Figure 4. (a) Field image of a sediment-clogged inlet located near Topeka, KS, USA, and (b) laboratory image from an experimental mix of clogging in a drain (with grate removed).

The sediment mixture consisted of modeling clay, $0.5 \mathrm{~mm}$ silica, and water with composition percentages of $30 \%, 46 \%$, and $24 \%$, respectively. The wet density of the mixture was $2.04 \mathrm{~g} / \mathrm{cm}^{3}$ and the dry density was $1.99 \mathrm{~g} / \mathrm{cm}^{3}$. The experiments were conducted as described in Figure 3 with three trials per combination. The sediment removal rate $(\mathrm{g} / \mathrm{s})$ was measured by recording the loss of sediment mass over the $10 \mathrm{~min}$ trials. Spread measurements were recorded at a location upstream and downstream of the inlet.

\subsubsection{Sediment Mixture Comparison}

Measured experimental erosion was contextualized within the literature by analyzing the relationship between dimensionless shear stress and transport. We present our results alongside a collection of natural mix types found in Walder [16]. For cohesive sediment, a dimensionless parameter for shear stress, $\phi$, and a dimensionless transport parameter, $R$, are defined as in Walder [16]:

$$
\begin{gathered}
\phi=\frac{\varepsilon}{\rho_{s}\left(\tau_{c} / \rho\right)^{0.5}} \\
\mathrm{R}=\frac{u_{*}^{2}-u_{c r}^{2}}{u_{c r}^{2}}
\end{gathered}
$$

where $\varepsilon=$ the sediment entrainment rate, $\rho_{s}=$ the density of the sediment, $\rho=$ the density of water, $\tau_{c}=$ the critical shear stress, $u_{*}=$ the shear velocity, and $u_{c r}=$ the critical shear velocity. Sediment entrainment was determined by van Rijn [17] as $\varepsilon=M / A t$ where $M=$ the total mass of sediment lost, $A=$ surface area, and $\Delta t=$ the measurement period. The shear velocity was determined as $u_{*}=\sqrt{g h S}$, where $g=$ gravity, $h=$ the hydraulic radius, and $S=$ the longitudinal slope. A critical shear stress of $0.014 \mathrm{~Pa}$ was used as determined from experimental testing of the upstream bed shear with a specific gravity of 2.04 and a dimensionless critical shear stress of 0.14 . Experimental erosion data by grate type were compared to meta-analysis by Walder [16] for a range of sediment types (Supplementary Materials Figure S1). The results of our created mixture were similar to other natural cohesive sediments as indicated in Walder [16]. 


\subsection{Precipitation Analysis}

To inform real-world decision making regarding optimal grate type as a function of flow regime, experimental results were applied to environmental conditions from eight sites across Illinois (IL) and Kansas (KS) by analyzing 15 min precipitation data over a 10-year period (2000-2010) [18]. The Kansas gauges were in the towns of Iola, Lawrence, Smolan, and Wilson and the Illinois gauges were in Chicago, Danville, Illinois City, and Quincy. The rainfall data were used to categorize actual events into three experimental inflow regimes (low, medium, and high). Cutoffs were determined through transforming the experimental inflow regimes of $0.7,1.0$, and $1.6 \mathrm{~m}^{3} / \mathrm{h}$ to rainfall intensities $(\mathrm{mm} / \mathrm{h})$ by dividing by the deck area resulting in equivalent intensities of 94,141 , and $220 \mathrm{~mm} / \mathrm{h}$ representing low, medium, and high intensities, respectively. Low-to-medium flow occurrences were defined as the number of events in the range of 51 to $220 \mathrm{~mm} / \mathrm{h}$ and high flow occurrences were events greater than $220 \mathrm{~mm} / \mathrm{h}$.

\subsection{Statistical Analysis}

Statistical analysis of data from both experiments were analyzed using a combination of IBM SPSS Statistics 27 software [19] and SciPy statistical package in Python [20]. All statistical tests used in this paper were performed with a significance level of $\alpha=0.05$. Statistical testing for this study incorporated all experimental trials. Results reflect all measurement error and variability; represented as the standard deviation within the results.

For the hydraulic efficiency experiment, our response variables were efficiency and spread. These results were non-normally distributed, as indicated by the Shapiro-Wilks's test; thus, hypothesis testing was performed using the non-parametric Mann-Whitney U-test [21]. One-Way Analysis of Variance (ANOVA) with the Tukey Post hoc test was used to examine the influence of inflow, longitudinal slope, grate type, downspout shape, and downspout size on the performance metrics of efficiency and spread. ANOVA is typically used to difference in means when more than two groups are present as in this study [22,23]. For the sediment removal experiment, the response variable was sediment removal rate. Distribution of erosion results was not normally distributed and statistical significance was evaluated with the Mann-Whitney U-test. One-Way ANOVA with the Tukey post hoc test was applied to analyze the influence of inflow, bridge deck slope, and grate type on sediment removal rate. Data were split by inflow regime to be consistent compared to the analysis of the hydraulic efficiency experiment.

\section{Results}

This section examines the results of the two experimental setups: hydraulic efficiency and sediment removal. These two results are explored within the context of three drivers: flow regime (a proxy for climate), bridge deck design, and inlet design. Significant experimental results are highlighted and discussed in each subsection.

\subsection{Climate: Inflow Regimes}

\subsubsection{Hydraulic Efficiency}

Efficiency and spread were compared for $6 \%$ cross slope only as measurements at $2 \%$ cross slope showed no response to discharge (Figure 5). The application of the one-way ANOVA indicated inflow regime significantly influenced hydraulic efficiency $\left(F_{2,285}=150.02, p<0.01\right)$. Analysis with the Tukey post hoc test indicated efficiency was significantly higher for the low flow condition $(\mu=0.93, \sigma=0.06)$ compared to the medium $(\mu=0.85, \sigma=0.09)$ and high $(\mu=0.69, \sigma=0.13)$ flow conditions. Inflow regime was also a significant influence $\left(F_{2,285}=154.92, p<0.01\right)$ on the spread of water into the roadway. Significant difference in average spread $(\mathrm{cm})$ was observed for all comparisons of low $(\mu=15.33, \sigma=1.82)$, medium $(\mu=17.57, \sigma=1.82)$, and high $(\mu=19.99, \sigma=1.87)$ inflow groupings. Our results indicate that for bridge decks with large cross slopes, the intensity of gutter flow, which we infer to be a proxy for precipitation intensity and climate, is a primary control on the performance of water capture efficiency and spread. 


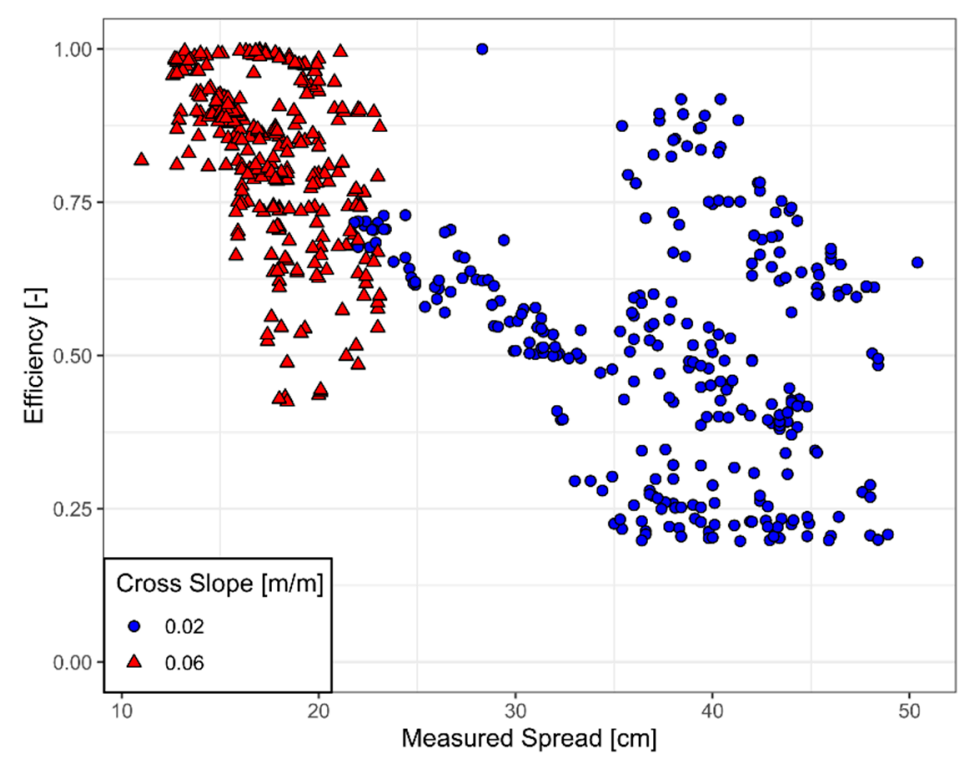

Figure 5. Cross slope comparison of experimental data for hydraulic efficiency compared to measured spread.

\subsubsection{Sediment Removal}

The magnitude of inflow significantly influenced the rate of sediment removal within drainage inlets $\left(F_{2,141}=3.93, p=0.02\right)$. Mean sediment removal rate $(\mathrm{g} / \mathrm{s})$ at the low inflow regime $(\mu=0.047, \sigma=0.019)$ were significantly different from erosion at the medium $(\mu=0.065, \sigma=0.045)$ and high $(\mu=0.063, \sigma=0.035)$ inflows. Surprisingly, no significant differences were observed between sediment removal rates at the medium and high inflow regimes, which we expected to occur given that high flows produce larger, more erosive shear stresses $[16,17]$. Potential explanations include non-linear erosion dynamics between flow rate and entrainment, uncertainty with mixture uniformity, measurement error, or the influence of other experimental variables as described below. Our results indicate that greater flow intensities, such as what may be experienced with climate change, cause reduced hydraulic efficiency of water capture; however, flow impact on sediment removal is less certain.

\subsection{Bridge Deck Design: Longitudinal and Cross Slopes 3.2.1. Hydraulic Efficiency}

The relationship between efficiency and spread was markedly different for the two cross slopes (Figure 5). At a $6 \%$ cross slope, efficiency co-varied with spread, whereas at the $2 \%$ cross slope, there was no consistent relation of efficiency in response to spread. The lack of response at $2 \%$ cross slope was attributed to a low energy condition, where friction or surface roughness may have controlled the transport of water downslope due to small flow depths [24]. With less gutter flow directly over the inlet openings, the amount of water removed from the opening is less-controlled by the inlet design $[25,26]$. The design implication of shallow cross slopes is that they perform poorly when it comes to channelizing roadway runoff into gutter flow, limiting the ability of drainage inlets to remove water, thus causing greater spread into the traffic lanes [27]. For shallow cross slopes, such as the $2 \%$ tested in this study, the measured spread is large, and the design of shoulder widths must consider whether they will be large enough to contain these high-water depths.

Across most experiments, hydraulic efficiency generally decreased as longitudinal slope increased (Figure 6). The exception to this observation was at the largest longitudinal slope where we observed an uptick in hydraulic efficiency. With respect to flow, hydraulic efficiency decreased as flow increased irrespective of longitudinal slope. This relationship was significant for all inflow rates: low $\left(F_{3,92}=102.64, p<0.01\right)$, medium $\left(F_{3,92}=21.17\right.$, 
$p<0.01)$, and high $\left(F_{3,92}=5.57, p<0.01\right)$. Regarding spread of water onto the roadway, we observed significantly lower spread as longitudinal slope increased (Figure 7). Irrespective of longitudinal slope, we found that spread significantly increased with increasing inflow across all flow regimes: low $\left(F_{3,92}=25.06, p<0.01\right)$, medium $\left(F_{3,92}=89.96, p<0.01\right)$, and high $\left(F_{3,92}=65.61, p<0.01\right)$.

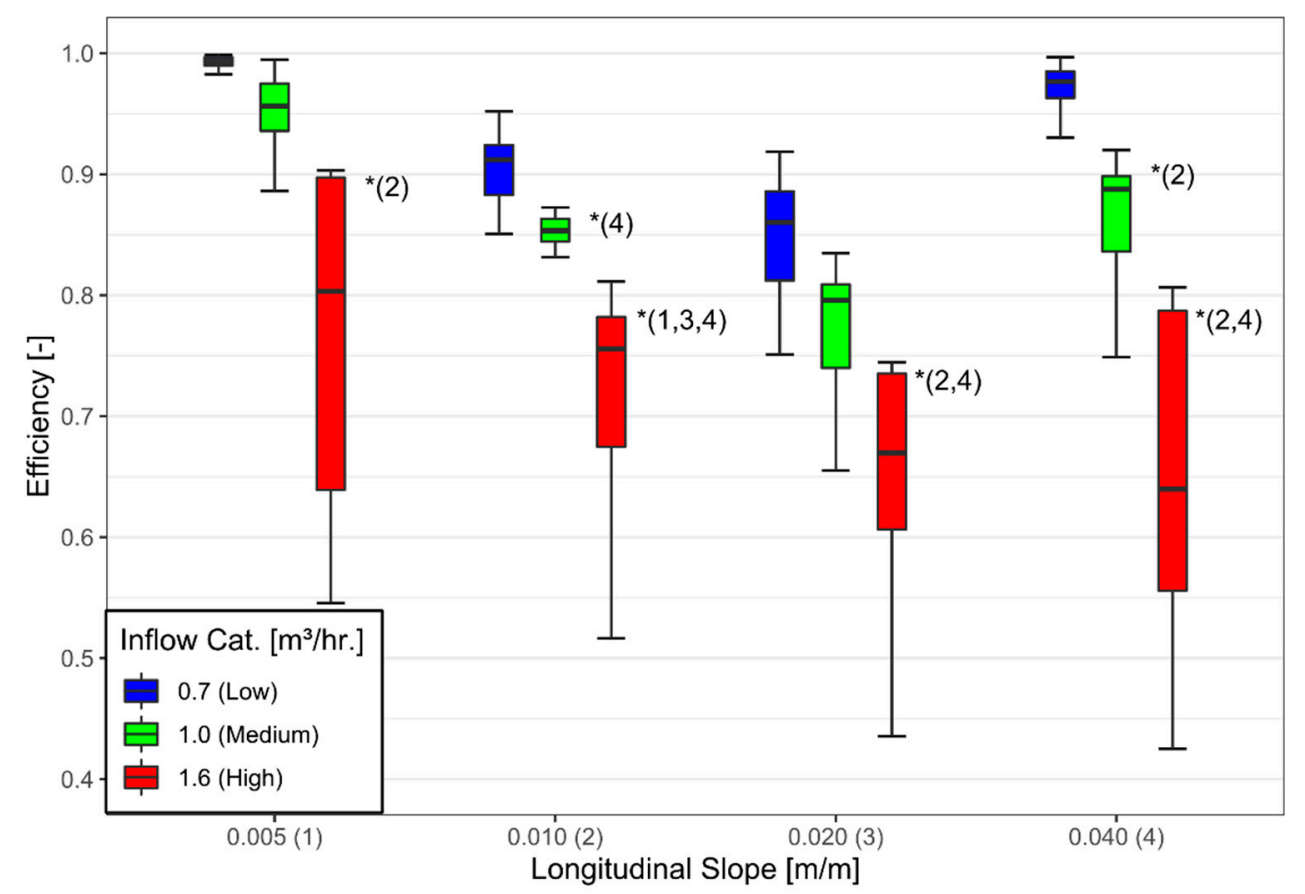

Figure 6. Efficiency boxplot based on inflow category comparing across longitudinal slope. (*) Indicates statistical similarity $(p>0.05)$ between means with numbers corresponding to opening size and shape given on the $\mathrm{x}$-axis.

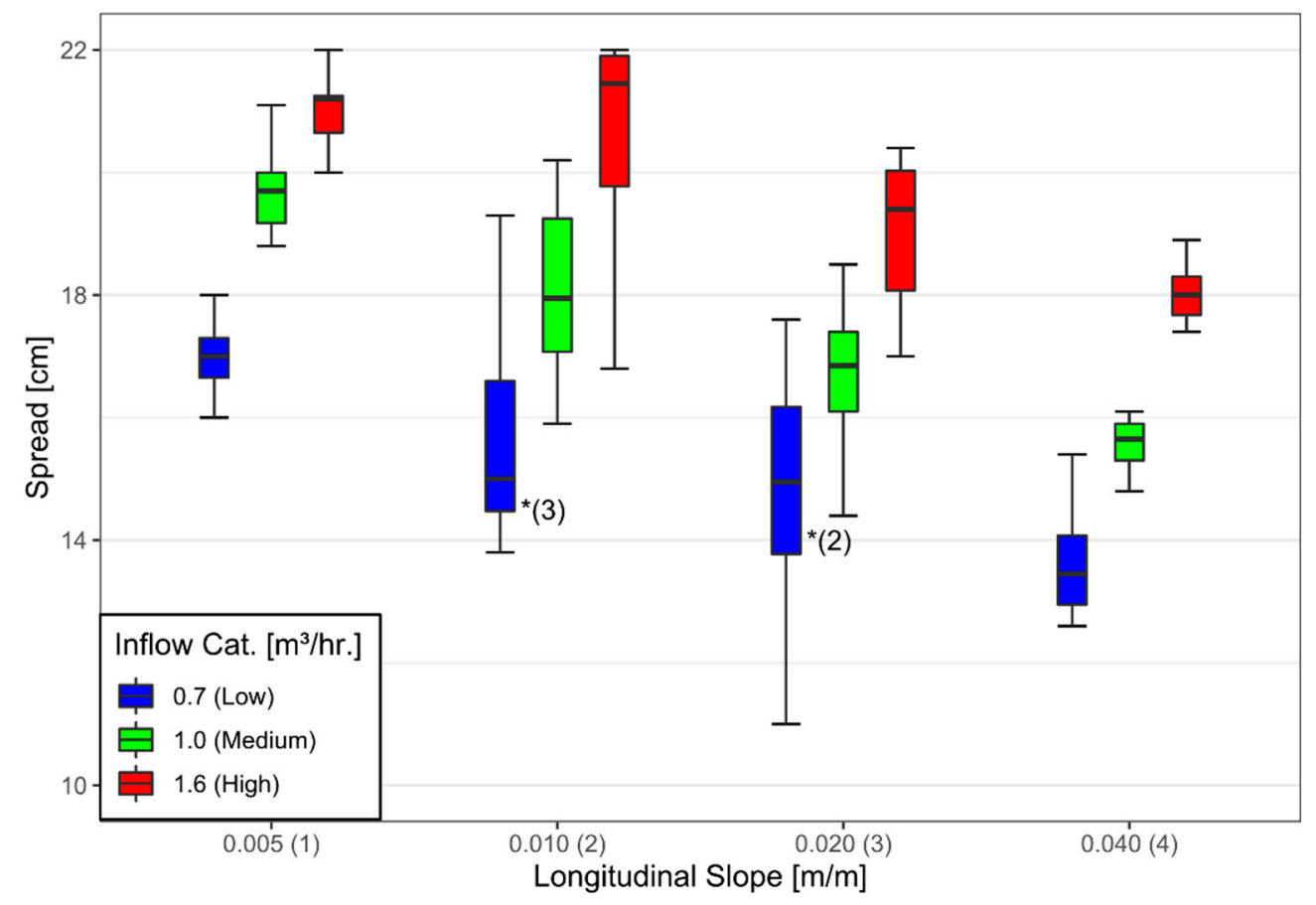

Figure 7. Spread measurement boxplot based on inflow category comparing across longitudinal slope. $\left(^{*}\right)$ Indicates statistical similarity $(p>0.05)$ between means with numbers corresponding to opening size and shape given on the $x$-axis. 
Overall, efficiency and spread co-varied for longitudinal slope across all inflow regimes, as shown in previous studies [2,25-27]. However, within our experiment the largest longitudinal slope produced an increase on average in efficiency for the low and medium inflow regimes (Figure 6) compared to the other studies in which the efficiency decreases as longitudinal slope increased. It is hypothesized that this is attributed to the placement of the inflow opening at the low end of the cross slope where it channelized water more than would be represented in the real world causing less flow bypass around the side of the inlet. These results confirm that the longitudinal slope of the bridge deck is a significant control on the overall performance of bridge deck drainage systems.

\subsubsection{Sediment Removal}

No statistical difference in mean sediment removal rates $(\mathrm{g} / \mathrm{s})$ between the cross slopes of $2 \%(\mu=0.054, \sigma=0.027)$ and $6 \%(\mu=0.063, \sigma=0.042)$ was observed. This was also true when statistical testing was applied within inflow regimes. The mean sediment removal rates at the $2 \%$ cross slope for low, medium, and high inflow were 0.045 $(\sigma=0.014), 0.058(\sigma=0.038)$, and $0.060(\sigma=0.024)$ compared to rates at $6 \%$ cross slope of $0.050(\sigma=0.023), 0.073(\sigma=0.050)$, and $0.066(\sigma=0.046)$. Uncertainties in our estimates range from 30 to $70 \%$, and thus no statistically significant differences could be determined. Increasing the number of experiments could help alleviate this issue. Despite this, cross slope should still be considered to have an impact on sediment scour as larger cross-slopes assist in greater channelization of gutter flow, larger longitudinal velocities, and higher shear stresses in the drainage inlet [28].

While longitudinal slope was not a significant influence on sediment removal rates for the low inflow regime $\left(F_{3,44}=5.57, p=0.78\right)$, it was significant at the medium $\left(F_{3,44}=6.04\right.$, $p<0.01)$ and high $\left(F_{3,44}=3.54, p=0.02\right)$ inflow regimes. For the medium inflow regime, mean sediment removal rates $(\mathrm{g} / \mathrm{s})$ at the steepest longitudinal slope of $4 \%(\mu=0.106$, $\sigma=0.070)$ were significantly different compared to the slopes of $0.5 \%(\mu=0.060, \sigma=0.027)$, $1 \%(\mu=0.046, \sigma=0.016)$, and $2 \%(\mu=0.050, \sigma=0.014)$. However, no significant differences in mean erosion were found comparing within the smaller slopes. The results for the longitudinal slope indicated that, on average, the sediment removal rates at a slope of $4 \%$ were $50 \%$ greater compared to the other three tested slopes. Since the flow would mainly be in the streamwise direction due to the steep slope gradient, a greater increase in bed shear over the sediment element occurs to remove particles from the inlet at a greater rate [29]. The use of steeper longitudinal slopes, when possible, could alleviate issues related to debris in bridge systems.

\subsection{Inlet Design: Size, Shape, and Grate Type}

\subsubsection{Hydraulic Efficiency}

Hydraulic efficiency was similar for both grate types (bar and vane) at low and medium flow regimes, but performance differed at the highest inflow rate. Average efficiency for the bar grate across low, medium, and high inflows was $0.93(\sigma=0.07), 0.86$ $(\sigma=0.07)$, and $0.74(\sigma=0.10)$, respectively, whereas vane grate values were $0.93(\sigma=0.06)$, $0.84(\sigma=0.09)$, and $0.65(\sigma=0.14)$, respectively. The bar grate design was significantly more efficient at the high inflow rate compared to the vane design. Statistical comparison of spread between grate types were similar with average values at low, medium, and high inflows of $15.28(\sigma=1.67), 17.54(\sigma=1.69)$, and $20.11(\sigma=1.86)$ for the bar grate and $15.38(\sigma=1.97), 17.59(\sigma=1.97)$, and $19.89(\sigma=1.90)$ for the vane grate, respectively. The larger vertical opening area of the bar grate compared to the vane rate allowed for greater flow interception at the high inflow regime explaining the difference in efficiency. This indicates that designers must consider grate type as a possible control on water drainage, particularly at high flows.

The downspout shape and width were also identified as controls on efficiency for medium and high flow, but not for low flow (Figure 8). For square downspouts, average efficiencies for low, medium, and high inflow were $0.94(\sigma=0.05), 0.87(\sigma=0.07)$, and 
$0.73(\sigma=0.11)$, respectively, whereas for circular downspouts they were $0.92(\sigma=0.07)$, $0.82(\sigma=0.09)$, and $0.66(\sigma=0.13)$, respectively. Similar relationships were observed for downspout widths $(20 \mathrm{~cm}$ vs. $25 \mathrm{~cm}$ ). Average efficiencies for $20 \mathrm{~cm}$ downspouts were 0.92 $(\sigma=0.07), 0.82(\sigma=0.09)$, and $0.61(\sigma=0.11)$ for low, medium, and high inflow, respectively, compared to $0.94(\sigma=0.06), 0.87(\sigma=0.07)$, and $0.73(\sigma=0.11)$ for $25 \mathrm{~cm}$ downspouts. In each case the design with the larger average cross-sectional area (square shape and/or $25 \mathrm{~cm}$ ), performed better indicating downspout area was an important control. At the high inflow regime, mean efficiency was significantly different between all opening areas (Figure 8). Application of larger downspout areas lessens the gap in efficiency between inflow regimes indicating that inclusions of larger opening areas in design would be beneficial for maintaining efficiency across all precipitation intensities.

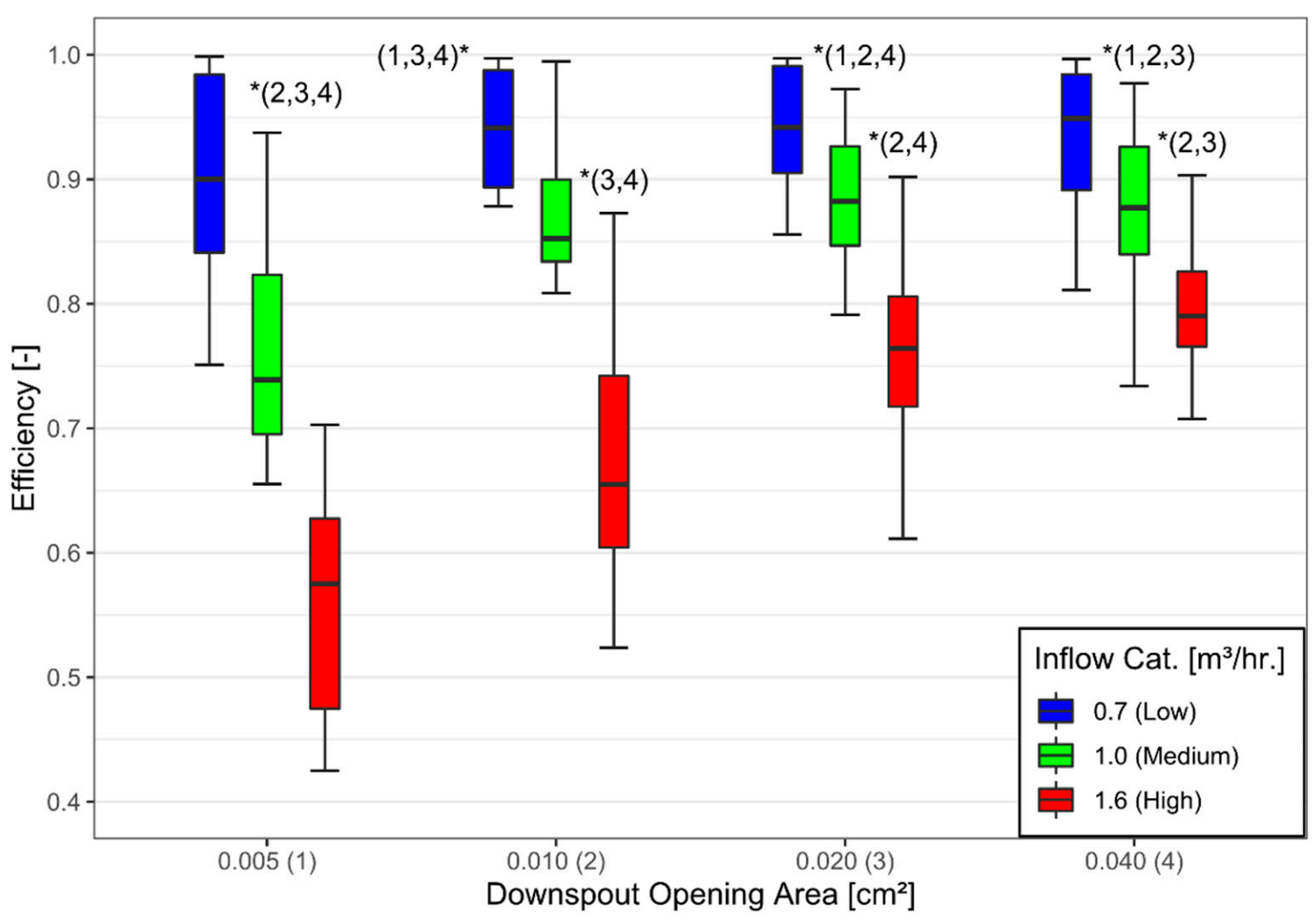

Figure 8. Efficiency boxplot based on inflow category comparing across downspout opening area. $\left({ }^{*}\right)$ Indicates statistical similarity $(p>0.05)$ between means with numbers corresponding to opening size and shape given on the $\mathrm{x}$-axis.

Downspout size and shape were a significant influence for the low $\left(F_{3,92}=10.67\right.$, $p<0.01)$, medium $\left(F_{3,92}=3.66, p=0.02\right)$, and high $\left(F_{3,92}=4.67, p<0.01\right)$ inflow regimes (Figure 9). For low inflow regimes, the smallest downspout area had significantly larger spreads compared to the three larger areas. For the medium inflow, spread gradually decreases as the opening area increases with significant difference between areas of 3.88 and $7.72 \mathrm{~cm}^{2}$. A transition region is likely occurring where the spread is partially controlled by downspout design, but the largest size alleviates the control condition. Similarly, for the high inflow regime in Figure 9, significant difference is only observed between the largest opening area and the two smallest sizes. Overall, the larger downspout design is beneficial to controlling spread across all conditions, and thus reducing the risk of hydroplaning.

\subsubsection{Sediment Removal}

Sediment removal rates between bar and vane grates differed based on inflow regime (Table 1). In general, sediment removal rate tends to increase with flow rate. This was true for all bar grate flow scenarios; however, this was not the case for vane grates as erosion 
increased initially from low to medium inflows, but then decreased at high flows (Table 1). No significant differences between grate types at each inflow regime were found, but we mainly attributed this to the small experimental sample size per inflow $(n=24)$.

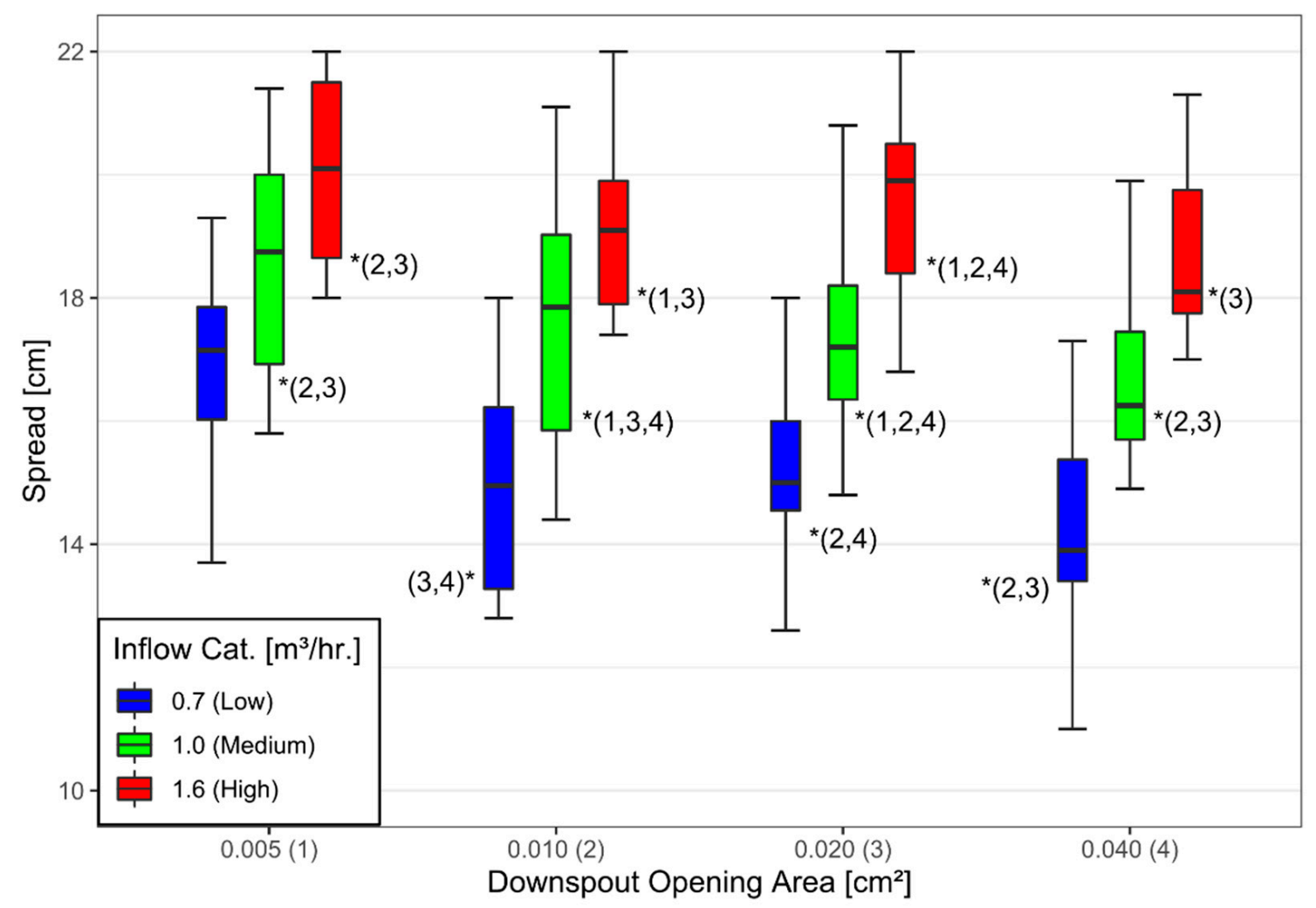

Figure 9. Spread measurement boxplot based on inflow category comparing across downspout opening area. ${ }^{*}$ ) Indicates statistical similarity $(p>0.05)$ between means with numbers corresponding to opening size and shape given on the $x$-axis.

Table 1. Sediment removal rates $( \pm 1 \sigma)$ by grate design and average inflow ( $n=24$ for each inflow).

\begin{tabular}{ccc}
\hline \multirow{2}{*}{$\begin{array}{c}\text { Inflow } \\
\left(\mathbf{m}^{\mathbf{3}} / \mathbf{h}\right)\end{array}$} & \multicolumn{2}{c}{ Sediment Removal Rate $\mathbf{( g / s )}$} \\
\cline { 2 - 3 } & Bar Grate & Vane Grate \\
\hline 0.7 & $0.045 \pm 0.015$ & $0.049 \pm 0.022$ \\
1.0 & $0.056 \pm 0.023$ & $0.075 \pm 0.059$ \\
1.6 & $0.067 \pm 0.045$ & $0.059 \pm 0.023$ \\
\hline
\end{tabular}

At low-to-medium flows, it is hypothesized that the vane grate is able to guide the water into the inlet as its design has curved bars, which transition from horizontal to vertical, aiding gravity in changing the direction of gutter flow to improve sediment removal rates. Observations from video recordings of the experiments found that, at low-to-medium flows, water could be seen traveling along the vane and diverted into the inlet along the vertical side of the cohesive sediment more efficiently compared to the bar grate that allowed more flow to bypass horizontally over the sediment material, supporting the results in Table 1 . However, at higher flows, when grates are submerged, it is hypothesized that the curvature of the vanes may cause an obstruction in the vertical flow path of water toward the downspout pipe, limiting erosion potential. Grate-splashover velocity relationships found in Johnson and Chang [30] and Brown et al. [31] support this idea as a vane grate was shown to have more splashover compared to a bar grate for large gutter flows. This explanation is plausible as the rectangular bar grate, with its bars parallel to potential vertical flow paths, had improved cleanout over the curved vane grate 
at high flows. The experiment shows how bridge configuration and inflow can affect the movement of debris based on grate type.

\section{Discussion}

\subsection{Controls and Implications}

Previous works on roadway drainage $[2,11-13,25,26]$ focus on the empirical relationship between efficiency and spread based on the controls tested in this study. We considered three types of controls for hydraulic performance of water and sediment removal: climate, bridge design, and drainage design to define the importance of each control for a more robust discussion. Inflow intensity, a proxy for climate, had significant impacts to hydraulic efficiency and spread for nearly all experiments, regardless of the evaluated parameter, e.g., longitudinal slope (Figures 6 and 7), and downspout area (Figures 8 and 9). However, inflow intensity is not a variable that can be controlled, but rather must be designed for while considering climate uncertainties. The two variables hydraulic engineers have some control over include bridge (cross and longitudinal deck slope) and drainage (grate and downspout configuration) design. Bridge design is typically not selected by hydraulic engineers, but rather by structural engineers. However, this variable was the primary control of measured spreads and efficiencies (Figure 5). In situations where structural engineers have flexibility with deck design, increased cross slope could help with improving hydraulic efficiency and reducing roadway spread. Our study indicates that low cross slopes drive the design based on surface roughness control of the water. Current literature is lacking in this regard as no known studies investigate this scenario indicating that intermediate cross slopes should also be investigated to understand the threshold in which this condition subsides. Additionally, longitudinal slope influenced design across all inflow regimes, which is consistent with previous studies $[2,13]$ and should be examined alongside cross slope when feasible. On the other hand, the shape and size of the downspout (drainage design) has significant performance implications and is specified by hydraulic engineers. We found that hydraulic engineers should choose square downspout rather than circular downspout designs. A square downspout of a given width will perform better, particularly during medium and high flows (Figures 8 and 9), as a circular design of an equivalent diameter, predominantly due to larger opening area despite having a similar installation footprint. Typically, related studies $[2,11,13,25,26]$ solely focus on a single inlet design, but we suggest further investigation should be coupled with our experimental results across more designs as various types are used in the practice.

Understanding the interactions between bridge deck design controls is important for designing safe and resilient drainage systems as there is the potential for increased rainfall intensities in the future due to climate change [4]. To illustrate, a study by Moustakis et al. [32] examined precipitation extremes and indicated that in the Midwest and Eastern United States high intensity storm events are expected to occur more frequently, i.e., what is now a 20 -year event will become a 7 -year event for $75 \%$ of the US. Designers must understand how their area will respond to future climate scenarios to ensure standard safety factors are met. Implementation of proactive design such as the examination of bridge slopes in the context of drainage and increasing the typical downspout size will help to account for these changes in rainfall intensity. Alongside implementing the design relationships found in this study, designers should also consider different measures for determination of design storm events. Currently, designs within the context of water engineering applies intensity-duration-frequency curves derived from series of annual maximum and partial duration series [6]. However, this approach relies on historical data to assess a return period based on stationarity [6]. A study by Mailhot and Duchesne [9] determined that due to climate change, stationarity is not satisfied. Instead, for the context of water resource design, they [6] proposed a simplified approach where a critical return period is based on design parameters to account for future climate extremes acting as a safety factor to increase the standard return period. Applying regional specific information with newer guidelines will allow for designers to account for future climate uncertainty. 
The results of our study not only provide strong justification for changes in drainage design but highlight important metrics to consider when analyzing current infrastructure resiliency and sustainability. Current literature [33] finds that poor road drainage systems have devastating impacts the economy, as structure failures due to poor drainage increasingly impact society through increased travel time and decrease roadway safety. Barreiro et al. (2021) [34] analyze the resilience to flooding scenarios in cities through 1-D and 2-D drainage models with respect to volume, flooding duration, flooded area, and affected infrastructure. Incorporation of our experimental results for cross slope, longitudinal slope, inflow, and inlet type can help better model these systems through portraying the important parameters for runoff removal. For instance, since inflow is an important parameter for drainage efficiency, our results would highlight that multiple simulation types for each inflow regime should be conducted to understand the resilience of systems. Additionally, our findings indicate how downspout configurations that impact drainage efficiency, highlight how models must incorporate the designs found in roadway infrastructure when examining flooding resilience for cities. Understanding the construction and design aspects of when a system was constructed is also crucial to understanding the impact of each system component on the sustainability of a network. Based on our findings, it is important to understand how inlet design and bridge deck design have changed overtime to be able to better understand where changes in roadway drainage need to be made to keep the drainage systems operating at necessary levels.

\subsection{Sediment-Precipitation Analysis}

One of the main applications of this work is that the choice of drainage design (i.e., grate and downspout specifications) impacts the flow and sediment dynamics, which are influenced by the geographic location of the bridge. Our results highlight this idea as sediment removal was shown to change based on grate type and inflow regime (Table 1). To illustrate this point, precipitation analysis across eight sites in Kansas and Illinois were used to select a recommended grate type based on expected storm intensities. Each site's unique storm intensity characteristics resulted in different recommendations for the type of grate to use in design. For the four sites in Kansas, events falling within the high-flow regime are the predominant storm types (Table 2), thus suggesting that bar grates are more suitable for effective design given that our experiments showed they performed better under these conditions (Table 1). For the Illinois sites of Chicago, Danville, and Illinois City, low-to-medium intensity events are temporally more common; thus, designers should utilize curved vane grates as they perform best under low and medium inflow rates. For Quincy, IL, the breakdown of events indicate that it would be advantageous to use the bar grate as it could more efficiently remove sediment as high intensity events are common. However, designers should note that surface runoff generation is directly associated with temporal patterns in rainfall intensity, which drives sediment yield through rainfall impact and scour [35]. It should also be noted that precipitation might not be the sole driver of sediment removal as sediment mixture is location dependent. Further investigation into debris-grate relationships should examine the impact of location specific soil type, land use, and traffic patterns to address other factors that may control erosion potential. Overall, with the creation of debris removal data, designers could easily integrate precipitation analysis coupled with climate change adjustment [6] to address issue of clogging in the field.

Further studies are needed to understand relationships between grate types and sediment transport to establish specific design guidance as well as examine future trends related to climate change. Sediment buildup and transport processes are highly variable with spatial heterogeneities across drainage areas [36], which need to be explored further for developed structures related to precipitation extremes. Along with determining sediment removal relationships, similar experiments can also be used to quantify a clogging coefficient to provide optimized safety factors for design based on debris patterns as performed by Guo and Mackenzie [37] and Gomez et al. (2019) [38]. This would allow designers to better predict field values of efficiency especially when coupled with specific 
design efficiency curves. Overall, accounting for debris removal would alleviate costs due to maintenance and bridge deck degradation, which can help to prolong bridge structures for the future and allow for better bridge performance $[39,40]$.

Table 2. Experimental intensity occurrence at 8-gauge locations for 2000-2010 from NOAA CDO precipitation data.

\begin{tabular}{cccccc}
\hline Location & $\begin{array}{c}\text { NOAA } \\
\text { Station ID }\end{array}$ & $\begin{array}{c}\text { Less than Test } \\
\text { Flow (\%) }\end{array}$ & $\begin{array}{c}\text { Low-to-Medium } \\
\text { Flow (\%) }\end{array}$ & $\begin{array}{c}\text { High Flow } \\
\text { (\%) }\end{array}$ & $\begin{array}{c}\text { Optimal Grate } \\
\text { Type }\end{array}$ \\
\hline Iola, KS, USA & COOP:143984 & 25.1 & 30.2 & 44.7 & Bar \\
Lawrence, KS, USA & COOP:141612 & 24.3 & 35.7 & 40.0 & Bar \\
Smolan, KS, USA & COOP:147551 & 24.6 & 33.0 & 42.4 & Bar \\
Wilson, KS, USA & COOP:148946 & 26.3 & 28.9 & 44.8 & Bar \\
Chicago, IL, USA & COOP:111577 & 29.8 & 37.1 & 33.1 & Vane \\
Danville, IL, USA & COOP:112140 & 17.1 & 51.5 & 31.4 & Vane \\
Illinois City, IL, USA & COOP:114355 & 21.5 & 40.5 & 38.0 & Vane \\
Quincy, IL, USA & COOP:117077 & 26.9 & 30.9 & 42.2 & Bar \\
\hline
\end{tabular}

\section{Conclusions}

In bridge deck drainage design, the primary goal is to reduce hydroplaning by removing runoff generated during storm events. Our results showed that downspout size and shape are the most practical design elements to improve flow removal, particularly during high flow rates. Incorporating larger-than-presently-necessary downspout designs is a simple solution that can combat expected future increases in precipitation intensity and frequency. With design of new infrastructure, the inclusion of hydraulic design within the structural and transportation design process could be important for future design policy as experiments results find that under low-medium inflow conditions design is controlled by bridge deck slope. Additionally, sediment removal experiments indicate that larger bridge deck slopes have more potential to erode debris build-up. Furthermore, curved vane grates had greater sediment cleanout rates at lower flow rates, whereas rectangular bar grates performed better at high inflows. Comparing the experimental inflows with rainfall over a ten-year period at eight sites showed how the distribution of precipitation events can be compared to help decide between grate types if debris removal performance is known. Finally, our data and analysis on controls of drainage efficiency and sediment removal rates provide researchers and practitioners guidance on metrics important to modeling roadway drainage, planning for future infrastructure needs, and assessing current resilience to flood of roadway structures.

The primary limitations identified in our study stem from our experimental model. The first limitation is we only examined two cross-slopes and four longitudinal slopes as model scale, materials, and available time did not allow to test more combinations of slopes. We recommend the testing of a wider range of cross slopes to determine when it becomes a primary control on intercepted flow compared to inflow. A greater density of longitudinal slopes should be tested to cover all available design conditions to better quantify the relationship between intercepted flow and spread width. Further analysis of sediment removal needs to be conducted to develop sediment removal design tables as well as examine future precipitation projections. We only analyzed a single cohesive mixture, but mixture types based on regional materials should be analyzed to understand how sediment removal rates relate to regional climates. Further research on debris in drainage inlets should couple sediment removal with sediment buildup to understand the process holistically. Overall, this study identifies the primary controls on the bridge deck drainage design through experimental results and analysis to facilitate proactive infrastructure upgrades in face of changing precipitation regimes.

Supplementary Materials: The following are available online at https:/ / www.mdpi.com/article/10 .3390/w13243556/s1, Figure S1: Dimensional erosion rate and transport stage relationship, Table S1: Bridge Deck Drainage Survey, Table S2: Breakdown of experimental model components. 
Author Contributions: Conceptualization, A.H., A.T.H., A.M. and J.R.; methodology, A.H., A.T.H. and A.M.; software, A.M.; validation, A.H., A.T.H. and J.R.; formal analysis, A.M.; investigation, A.H., A.T.H., A.M. and J.R.; resources, J.R.; data curation, A.M.; writing—original draft preparation, A.M.; writing-review and editing, A.H., A.T.H. and J.R.; visualization, A.H., A.T.H., A.M. and J.R.; supervision, J.R.; project administration, J.R.; funding acquisition, J.R. All authors have read and agreed to the published version of the manuscript.

Funding: The authors would like to acknowledge the Kansas Department of Transportation Award KTRAN:KU-20-1 and the University of Kansas Department of Civil, Environmental, and Architectural Engineering for funding on this project.

Institutional Review Board Statement: Not applicable.

Informed Consent Statement: Not applicable.

Data Availability Statement: The data presented in this study are available in the Supplementary Material of the online version of this article.

Acknowledgments: We thank two anonymous reviewers for their constructive feedback, which improved the quality of this manuscript. We would like to thank Mike Orth at the Kansas Department of Transportation for his guidance throughout this project. Additionally, we thank Tim Craven at the Illinois Department of Transportation for his insight and providing the curved vane grate detail used for this study. Finally, the following provided help for construction of the scale model: Kent Dye, Josh Ostermann, and Amirreza Zarnaghsh.

Conflicts of Interest: The authors declare no conflict of interest.

\section{References}

1. Khasnabis, S.; Dhingra, S.L.; Mishra, S.; Safi, C. Mechanisms for transportation infrastructure investment in developing countries. J. Urban. Plann Dev. 2010, 136, 94-103. [CrossRef]

2. Qian, Q.; Liu, X.; Barret, M.E.; Charbeneau, R.J. Physical modeling on hydraulic performance of rectangular bridge deck drains. Water 2016, 8, 67. [CrossRef]

3. ASCE. 2021 Report Card for America's Infrastructure; American Society of Engineers: Reston, VA, USA, 2021.

4. IPCC. Climate Change. Synthesis Report; Intergovernmental Panel on Climate Change (IPCC): Geneva, Switzerland, 2014.

5. Kim, K.; Ha, S.; Kim, H. Using real options for urban infrastructure adaptation under climate change. J. Clean. Prod. 2017, 43, 40-50. [CrossRef]

6. Mailhot, A.; Duchesne, S. Design criteria of urban drainage infrastructures under climate change. J. Water Resour. Pann. Manag. 2010, 136, 201-208. [CrossRef]

7. Schweikert, A.; Chinowsky, P.; Kwiatkowski, K.; Espinet, X. The infrastructure planning support system: Analyzing the impact of climate change on road infrastructure and development. Transp. Policy 2014, 35, 146-153. [CrossRef]

8. Hu, S.; Zhi-mao, G.; Jun-ping, Y. The impacts of urbanization on soil erosion in the Loess Plateau region. J. Geogr. Sci. 2001, 11, 282-290. [CrossRef]

9. Fletcher, S.; Lickley, M.; Strzepek, K. Learning about climate change uncertainty enables flexible water infrastructure planning. Nat. Commun. 2019, 10, 1782. [CrossRef]

10. Young, G.K.; Walker, S.E.; Chang, F. Design of Bridge Deck Drainage; FHWA-SA-92-010, Hydraulic Engineering Circular 21 (HEC 21); Federal Highway Administration (FHWA): Washington, DC, USA, 1993.

11. Izzard, C.F. Hydraulics of runoff from developed surfaces. In Proceedings of the 26th Annual Meeting of Highway Research Board National Research Council, Washington, DC, USA, 5-8 December 1946; pp. 129-150.

12. Izzard, C.F. Tentative Results on Capacity of Curb Opening Inlet; Research Report No. 11-B.; Highway Research Board: Washington, DC, USA, 1950; pp. 36-51.

13. Schalla, F.E.; Ashraf, M.; Barrett, M.E. Limitations of traditional capacity equations for long curb inlets. Transp. Res. Rec. 2017, 2638, 97-103. [CrossRef]

14. Kansas Department of Transportation. Design Manual Volume III—Bridge Section; Kansas Department of Transportation (KDOT): Topkeka, KS, USA, 2015.

15. Illinois Department of Transportation. Bridge Manual; Illinois Department of Transporation (ILDOT): Springfield, IL, USA, 2012.

16. Walder, J.S. Dimensionless Erosion Laws for Cohesive sediment. J. Hydraul. Eng. 2015, 142. [CrossRef]

17. Van Rijn, L.C. Sediment pick-up functions. J. Hydraul. Eng. 1984, 110, 1494-1502. [CrossRef]

18. National Oceanic and Atmospheric Administration. National Centers for Environmental Information Data Available on the World Wide Web. NOAA Climate Data Online. Available online: https://www.ncdc.noaa.gov/cdo-web/ (accessed on 4 February 2021).

19. IBM Corp. IBM SPSS Statistics for Windows; Version 27.0; IBM Corp: Armonk, NY, USA, 2020.

20. Virtanen, P.; Gommers, R.; Oliphant, T.E.; Haberland, M.; Reddy, T.; Cournapeau, D.; Burovski, E.; Peterson, P.; Weckesser, W.; Bright, J.; et al. SciPy 1.0: Fundamental algorithms for scientific computing in python. Nat. Methods 2020, 17, 261-272. [CrossRef] 
21. Wilk, D.S. Statistical Methods in the Atmospheric Sciences, 3rd ed.; Academic Press: Oxford, UK, 2011; ISBN 978-0-12-3850255-5.

22. Wang, F.; Huang, G.H.; Fan, Y.; Li, Y.P. Robust Subsampling ANOVA methods for sensitivity analysis of water resource and environmental models. Water Resour. Manag. 2020, 34, 3199-3217. [CrossRef]

23. Blanca, M.J.; Alarcón, R.; Arnau, J.; Bono, R.; Bendayan, R. Non-Normal data: Is ANOVA still a valid option? Psicothema 2017, 29, 552-557. [CrossRef]

24. Zhang, S.; Liu, Y. Experimental study on anisotropic attributes of surface roughness in watersheds. J. Hydrol. Eng. 2017, 22, 06017005. [CrossRef]

25. Holley, E.R.; Woodward, C.; Brigneti, A.; Ott, C. Hydraulic Characteristics of Recessed Curb Inlets and Bridge Drains; Rep. No. FHWA/TX-92/0-1267-2F; Center for Transportation Research, the University of Texas at Austin: Austin, TX, USA, 1992.

26. Hammons, M.A.; Holley, E.R. Hydraulic Characteristics of Flush Depressed Curb Inlets and Bridge Deck Drains; Rep. No. FHWA/TX95/0-1409-01; Center for Transportation Research, the University of Texas at Austin: Austin, TX, USA, 1995.

27. Hermange, C.; Todoroff, V.; Biesse, F.; Le-Chenadec, Y. Experimental investigation of the leading parameters influencing the hydroplaning phenomenon. Veh. Syst. Dyn. 2021, 1-18. [CrossRef]

28. Kinnell, P.I.A. The influence of time and other factors on soil loss produced by rain-impacted flow under artificial rainfall. $J$. Hydrol. 2020, 587, 125004. [CrossRef]

29. Cao, L.; Zhang, K.; Zhang, W. Detachment of road surface soil by flowing water. Catena 2009, 76, 155-162. [CrossRef]

30. Johnson, F.L.; Chang, F. Drainage of Highway Pavement; Hydraulic Engineering Circular No. 12 (HEC-12); Federal Highway Administration (FHWA): Washington, DC, USA, 1984.

31. Brown, S.A.; Schall, J.D.; Morris, J.L.; Doherty, C.L.; Stein, S.M.; Warner, J.C. Urban Drainage Design Manual: FHWA-NHI-10-009, Hydraulic Engineering Circular 22 (HEC-22), 3rd ed.; Federal Highway Administration (FHWA): Washington, DC, USA, 2009.

32. Moustakis, Y.; Papalexiou, S.M.; Onof, C.J.; Paschalis, A. Seasonality, intensity, and duration of rainfall extremes change in a warmer climate. Earth's Future 2021, 9, 1-15. [CrossRef]

33. Awwad, M. Studying the effects of roads geometry and design parameters on the pavement drainage system. Civ. Eng. J. 2021, 7, 49-58. [CrossRef]

34. Barreiro, J.; Lopes, R.; Ferreira, F.; Matos, J.S. Index-Based approach to evaluate city resilience in flooding scenarios. Civ. Eng. J. 2021, 7, 197-207. [CrossRef]

35. Tao, W.; Wu, J.; Wang, Q. Mathematical model of sediment and solute transport along slope land in different rainfall pattern conditions. Sci. Rep. 2017, 7, 44082. [CrossRef]

36. Naves, J.; Anta, J.; Suarez, J.; Puertas, J. Hydraulic, wash-off and sediment transport experiments in a full-scale urban drainage physical model. Sci. Data. 2020, 7, 44. [CrossRef] [PubMed]

37. Guo, J.; Mackenzie, K. Hydraulic Efficiency of Grate and Curb-Opening Inlets under Clogging Effect; Research Report CDOT-2012-3; DTD Applied Research and Innovation Branch, Colorado Department of Transportation (CDOT): Denver, CO, USA, 2012.

38. Gomez, M.; Pares, J.; Russo, B.; Martinez-Gomariz, E. Methodology to quantify clogging coefficients for grated inlets. Application to SANT MARTI catchment. J. Flood Risk Manag. 2019, 12, e12479. [CrossRef]

39. Ghodoosi, F.; Abu-Samra, S.; Zeynalian, M.; Zayed, T. Maintenance cost optimization for bridge structures using system reliability analysis and genetic algorithms. J. Constr. Eng. Manag. 2018, 144, 04017116. [CrossRef]

40. Kim, K.H.; Nam, M.S.; Hwang, H.H.; Ann, K.Y. Prediction of remaining life for bridge decks considering deterioration factors and propose of prioritization process for bridge deck maintenance. Sustainability 2020, 12, 10625. [CrossRef] 